\title{
PLANIFICACIÓN ESTRATÉGICA EN EMPRESAS DEL SECTOR PÚBLICO DE LA PROVINCIA DE SANTA ELENA, 2017
}

\section{STRATEGIC PLANNING IN COMPANIES OF THE PUBLIC SECTOR IN THE PROVINCE OF SANTA ELENA 2017}

\author{
Jacqueline Bacilio Bejeguen ${ }^{{ }^{*}}$ \\ Lorena Reyes Tomalá ${ }^{1}$ \\ Mario Alomoto Tomalá 1 \\ Luis De la Cruz Pozo' \\ María Salgado Suárez ${ }^{1}$ \\ Jacinto Sánchez Lino ${ }^{1}$ \\ 1'Universidad Estatal Península de Santa Elena, Ecuador. *ibacilio@upse.edu.ec
}

\section{RESUMEN}

El objetivo del presente artículo consiste en diagnosticar la función administrativa de las instituciones públicas de la provincia de Santa Elena, específicamente la etapa de la planificación estratégica, la contribución teórica se realiza con la finalidad de confrontar la parte teórica con la práctica, de reconocer que la planificación estratégica tiene su razón de ser, como es la satisfacción de las necesidades de la población y velar por la calidad del servicio prestado; y, que se ejecuta a través de un proceso participativo y continuo. Se exponen los resultados de las encuestas realizadas a los administradores de las instituciones públicas de la provincia de Santa Elena que formaron parte de una muestra simple pero que realzan la importancia de esta función y explica las debilidades, que como ciudadanos visualizamos al reclamar nuestro derecho como contribuyentes, dejando en el pensamiento si para ser un administrador público necesita conocimientos y habilidades más que los votos populares o una simple designación.

Palabras claves: administración, función, planificación estratégica, instituciones públicas.

\begin{abstract}
The aim of this article is to diagnose the administrative function of the public institutions of the province of Santa Elena, specifically the stage of strategic planning, the theoretical contribution is made with the purpose of confronting the theory with practice, to recognize that the strategic planning has its reason to be, as is the satisfaction of the needs of the population and ensure the quality of the service rendered; and, that is executed through a participatory and continuous process. The results of the surveys directed to administrators of public institutions of the province of Santa Elena who formed part of a simple sample but that highlight the importance of this function and explains the weaknesses, that we as citizens visualize to claim our right as taxpayers, leaving in the thinking if a public administrator needs knowledge and skills more than the popular votes or a simple designation.
\end{abstract}

Keywords: administration, function, strategic planning, public institutions.

Recibido: 07 de marzo de 2018

Aprobado: 07 de mayo de 2018

Publicado: 20 de junio de 2018 


\section{INTRODUCCIÓN}

La palabra gestión ha evolucionado a partir de que las funciones de los gerentes 0 administradores de empresas, se han vuelto más exigentes con la finalidad de mejorar el desempeño institucional a través de sus funciones; la gestión nos permite resolver objetivos planteados mediante técnicas de seguimiento, optimización de recursos, pronósticos, entre otros, con el propósito de alcanzarlos en el tiempo propuesto y también según los acontecimientos permitidos.

El presente trabajo tiene como objetivo revisar definiciones respecto a gestión, gestión estratégica y pública que permita la recopilación de información en las instituciones públicas de la Provincia de Santa Elena para comparar la práctica con la teoría y realizar conclusiones para posteriores investigaciones.

Desde la era de la globalización, la tecnología y el conocimiento las funciones administrativas públicas dejan de ser actividades detrás de un escritorio y ejecutadas por personas formadas para desempeñar estas funciones, sino que son ocupaciones que requieren de un alto desarrollo de habilidades técnicas, de comunicación y cognitivas sin ningún grado de prioridad, estas se armonizan y se ejecutan de acuerdo a las situaciones cotidianas.

\section{Gestión}

Según Jiménez (2014) en su obra define a la palabra gestión como guías para orientar la acción, previsión, visualización y empleo de los recursos y esfuerzos a los fines que se desean alcanzar, la secuencia de actividades que habrán de realizarse para lograr los objetivos y el tiempo requerido para efectuar cada una de sus partes y de todos aquellos eventos involucrados en su concesión. (p. 290).

La Gestión Pública (Jiménez, 2014) sugiere definirla como el "Conjunto de acciones mediante las cuales las entidades tienden al logro de sus fines, objetivos y metas, los que están enmarcados por las políticas gubernamentales establecidas por el Poder Ejecutivo" (p.293). La gestión pública nos ayuda a realizar una actividad de manera correcta pero en el sector de empresas estatales considerando las empresas públicas o departamentos de gobierno.

La Gestión estratégica según el mismo autor (Jiménez, 2014) establece que es la determinación de los fines y objetivos a largo plazo de la empresa, junto con la adopción de cursos de acción y la asignación de recursos necesarios para lograr estos objetivos. (p.293).
La gestión estratégica es la que nos permite alcanzar nuestros objetivos en la empresa teniendo una excelente administración y preocupándose por el cliente en la entrega de calidad del servicio público y siendo competencia con las demás instituciones gubernamentales generando un mercado competitivo y beneficioso para el usuario, consiste en el proceso de definición (hoy) de lo que queremos ser en el futuro, apoyando en la correspondiente reflexión y pensamiento estratégico.

El plan estratégico constituye la herramienta en la que la alta dirección recorre las decisiones estratégicas corporativas que ha adoptado $<<$ hoy $>$ > (es decir, en el momento que ha realizado la reflexión estratégica con su equipo de dirección), en referencia a lo que hará en los tres próximos años, para lograr una empresa competitiva que le permite satisfacer las expectativas de sus diferentes grupos de interés. (Sainz, 2015)

\section{El sector público}

Según (Jiménez, 2014). Conjunto de actividades económicas que están bajo el control del Estado. En Cuentas Nacionales, se refiere a parte del sistema económico que está relacionado con la actividad estatal, ya sea financiera $o$ no financiera. Incluye a las entidades pertenecientes al gobierno central e instancias descentralizadas así como a las empresas públicas financieras y no financieras que proveen bienes y/o servicios. El sector público está conformado por diferentes instituciones afines al estado, esto quiere decir, que las organizaciones deben estar enmarcadas a las políticas públicas del poder estatal. Las organizaciones que tienen estos rubros aportan al estado con los bienes y servicios brindados a la comunidad, por otro lado hay instituciones las cuales no son financieras pero que de todas maneras aportan a la sociedad.

Se llama sector público al conjunto que forman las corporaciones, las instituciones y las oficinas del Estado. Debido a que el Estado es una forma de organización de la sociedad, se entiende que aquello que es estatal es público, en el sentido de que no pertenece a una persona o una empresa sino que es propiedad de todos los habitantes. (Pérez \& Gardey, 2014). Una empresa del sector público podríamos denominarla como una institución del estado, el cual presta un producto o servicio a su comunidad, tienen una gran diferencia con las empresas privadas puesto que las públicas están más acorde a las necesidades de la comunidad en ámbito de situación económica. 


\section{Quienes son las empresas del sector público}

Las empresas del sector público pueden ser beneficiarias de subvenciones en los mismos términos que lo pudiera ser cualquier empresa perteneciente al sector privado, el objetivo de imagen fiel exige excepcionar en estos casos (subvenciones otorgadas a empresas públicas por sus socios para financiar la realización de actividades de interés público o general. (Sergio, 2017). El sector público están conformadas por empresa pública y entidades gubernamentales la cuales están precedidas por un gerente que es el encargado de administrarla y cumplir sus objetivos y metas; no obstante dejar de lado la políticas del poder ejecutivo al cual deben estar enmarcadas las empresas del sector público.

\section{Administración pública del Ecuador}

Según (Tobar) La administración pública del Ecuador ha evolucionado a través de los años realizando mejoras en los reglamentos para los beneficios de los empleados públicos, en el año 1923 se creó la Caja de Pensiones, para dar protección social en particular a los empleados públicos. En el año 1928 se promulgo la ley Orgánica de Haciendas, para regular sus nombramientos, salarios y responsabilidad de los servidores públicos. En 1959 se publica la ley de Carrera administrativa que establece: La estabilidad de funcionarios públicos; su capacitación técnica; y su sistema de selección. En 1968, la ley de Servicio Civil y Carrera administrativa establece; un sistema de administración del personal, basado en merito; igual oportunidad, sin discriminación para todos los ecuatorianos hasta la actualidad.

Tabla 1. Población de provincia de Santa Elena

\begin{tabular}{llllll}
\hline Cantones & Hombre & \multicolumn{3}{c}{ Mujeres } & Total \\
\hline La Libertad & 48.030 & $30.6 \%$ & 47.912 & $31.6 \%$ & 95.942 \\
Salinas & 35.436 & $22.6 \%$ & 33.239 & $21.9 \%$ & 68.675 \\
Santa Elena & 73.396 & $46.8 \%$ & 70.680 & $46.6 \%$ & 144.076 \\
Total & 156.862 & $100 \%$ & 151.831 & $100 \%$ & 308.693 \\
\hline
\end{tabular}

Realizado por (INEC), 2010)

\section{Administración de Pública en la Provincia de Santa Elena}

La provincia de Santa Elena es la número de 24 del Ecuador y consta de 3 cantones y contiene según el Instituto Nacional Estadística y Censo 308.693 habitantes que están distribuidos entre hombres y mujeres por cada cantón de la siguiente manera: (INEC), 2010)

El $14 \%$ de la población Santa Elena trabaja en instituciones públicas según el INEC en su último censo lo que es muy probable que el porcentaje subiera en los siguientes años.

Tabla 2. Ocupación de los habitantes de Santa Elena

\begin{tabular}{|l|c|c|c|}
\hline Ocupación & Hombre & Mujer & Habitantes \\
\hline Empleado Privado & 21.539 & 6.386 & 27.925 \\
\hline Cuenta propia & 21.433 & 7.588 & 29.021 \\
\hline Jornalero o peon & 13.047 & 553 & 13.600 \\
\hline Empleado u obrero del Estado, Municipio o Consejo Provincial & 8.379 & 4.259 & 12.638 \\
\hline No declarado & 3.475 & 2.043 & 5.518 \\
\hline
\end{tabular}

Tabla diseñada con datos del (INEC), 2010)

La empresa pública en la provincia de Santa Elena tuvo apertura según (Marjorie Rodríguez C., 2016) a partir de la Creación del Cantón Santa Elena, porque nace el Gobierno Autónomo Descentralizado Municipal de Santa Elena y sus respectivas autoridades. La creación del Gobierno Autónomo Descentralizado Municipal de Santa Elena se fundamenta en el siguiente Marco Legal:
- Constitución 2008 - Ecuador.

- Ley Orgánica del Servicio Público.

- Código Orgánico de Organización

- Territorial, Autonomía y Descentralización

- Código del trabajo

- Ley Orgánica del Consejo de Participación Ciudadana y Control Social

- Código Orgánico de Planificación y Finanzas Públicas 
- Normas de Control Interno y

- Reglamento interno de la Institución.

Por lo tanto hoy en día la única empresa gubernamental no es solo el municipio de Santa Elena, sino que la provincia de Santa Elena en el sector público cuenta con 44 instituciones gubernamentales las cuales en este trabajo analizaremos la gestión estratégica que ejercen.

\section{Materiales y métodos}

Para lograr los fines de la investigación se realizó una investigación exploratoria dirigida a los administradores de las instituciones públicas de la provincia de Santa Elena a través de encuestas en los meses de julio, agosto y septiembre del año 2017 utilizando un muestreo probabilístico simple, la fracción muestral fue de 40 instituciones públicas.

El objetivo de las encuestas fue reconocer el entorno de trabajo de los gerentes, actividades que ejecutan, focalizada en la tendencia actual del servicio público.

\section{Resultados}

\section{Funciones de gestión administrativa}

De las encuestas realizadas a las instituciones públicas de la provincia de Santa Elena con respecto a qué fase utilizan para la gestión empresarial se obtuvo que un $75 \%$ planifica, organiza, dirige y controla, mientras que un $15 \%$ planea, organiza y dirige y el $10 \%$ que planea, controla y organiza. Estos valores indican que el $25 \%$ de las instituciones no consideran una función importante el control y la dirección.

\section{Planificación}

Figura 1. Gestión Administrativa

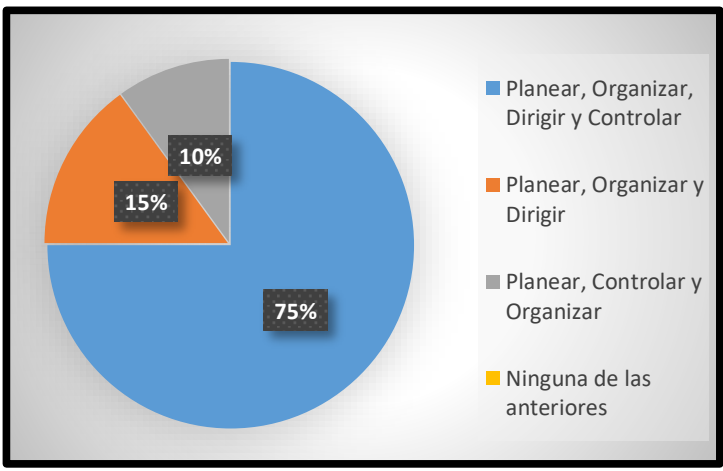

Fuente: Autores.

Insumos: la mayoría de las empresas públicas utilizan como insumo el diagnóstico institucional, misión y visión, presupuestos, dando menos importancia a los informes de años anteriores y el análisis de las necesidades externas como usuarios y proveedores.

Figura 2. Insumos de planificación

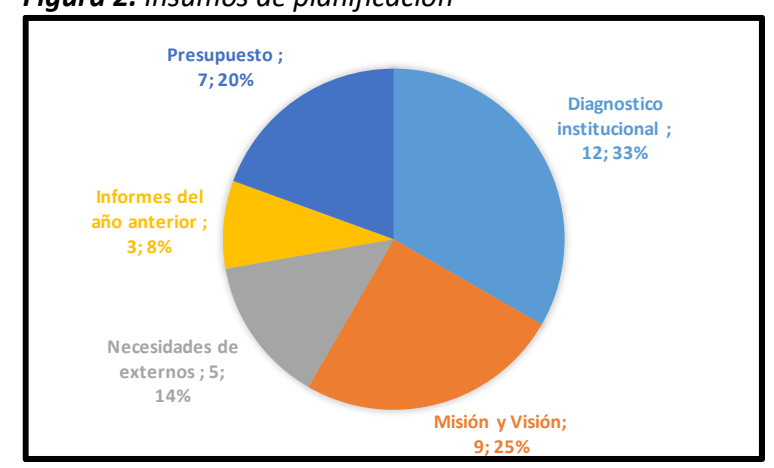

Fuente: Autores.

\section{Vigencia e importancia}

El $96 \%$ de las instituciones contestaron que su planificación estratégica tiene vigencia un año, mientras que un $2 \% 6$ meses y $1 \%$ entre 2 y 5 años respectivamente; consideran importante la planificación por las siguientes razones:

* Se define actividades a realizar

* Permite coordinar el trabajo

* Se establece presupuesto y personal

* Mejora los procesos y optimiza recursos

* Permite respetar el tiempo de los demás

* Se obtienen buenos resultados

* Permite adelantar y estar preparados antes sucesos

El tiempo ideal de la planificación según la experiencia de los encuestados, el $46 \%$ sostuvieron que el tiempo ideal es anual, el $44 \%$ mensual y el $10 \%$ semestral según su criterio personal.

Figura 3. Experiencia en Panificación

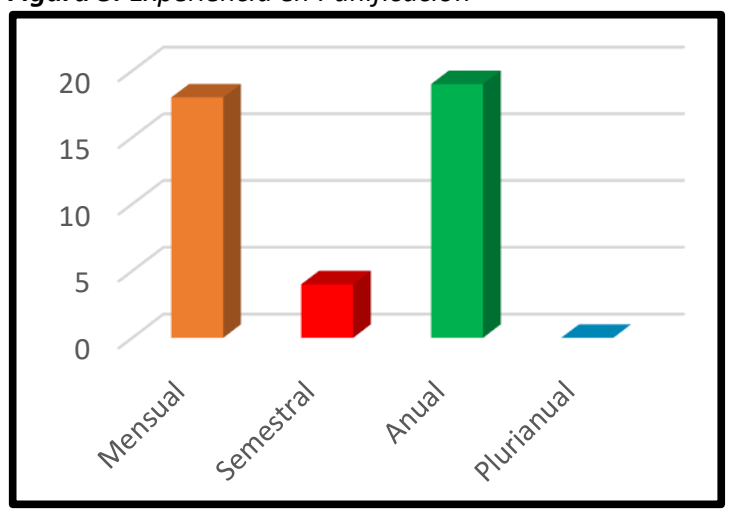

Fuente: Autores. 
Figura 4. Fines de la planificación estratégica

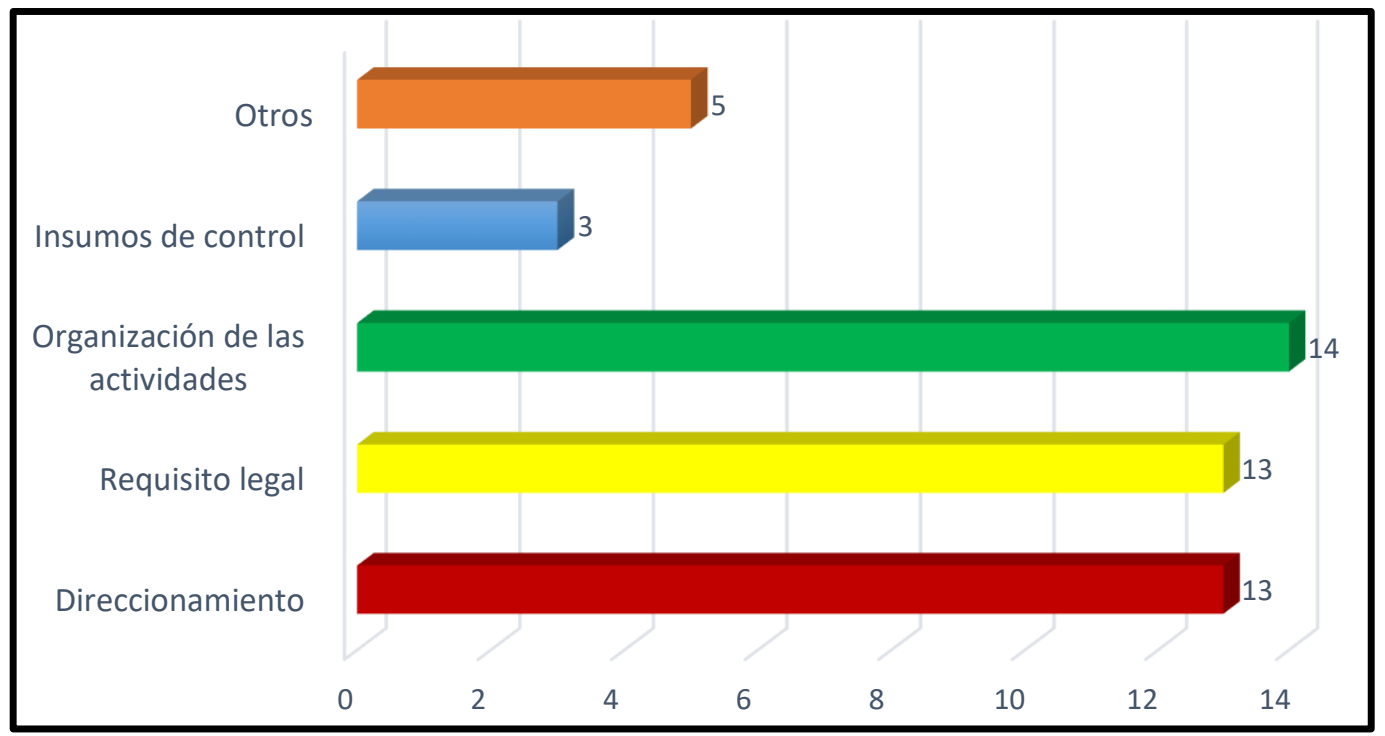

Fuente: Autores.

\section{Fines de la planificación estratégica}

La mayoría de las empresas manifestaron que realizan la planificación por la organización de las actividades así como también por dar un direccionamiento a la empresa y cumplir el requisito legal mientras que 3 instituciones mencionaron que lo hacen por ser un insumo para el control y 5 empresas que tienen otros fines.

Figura 5. Fines

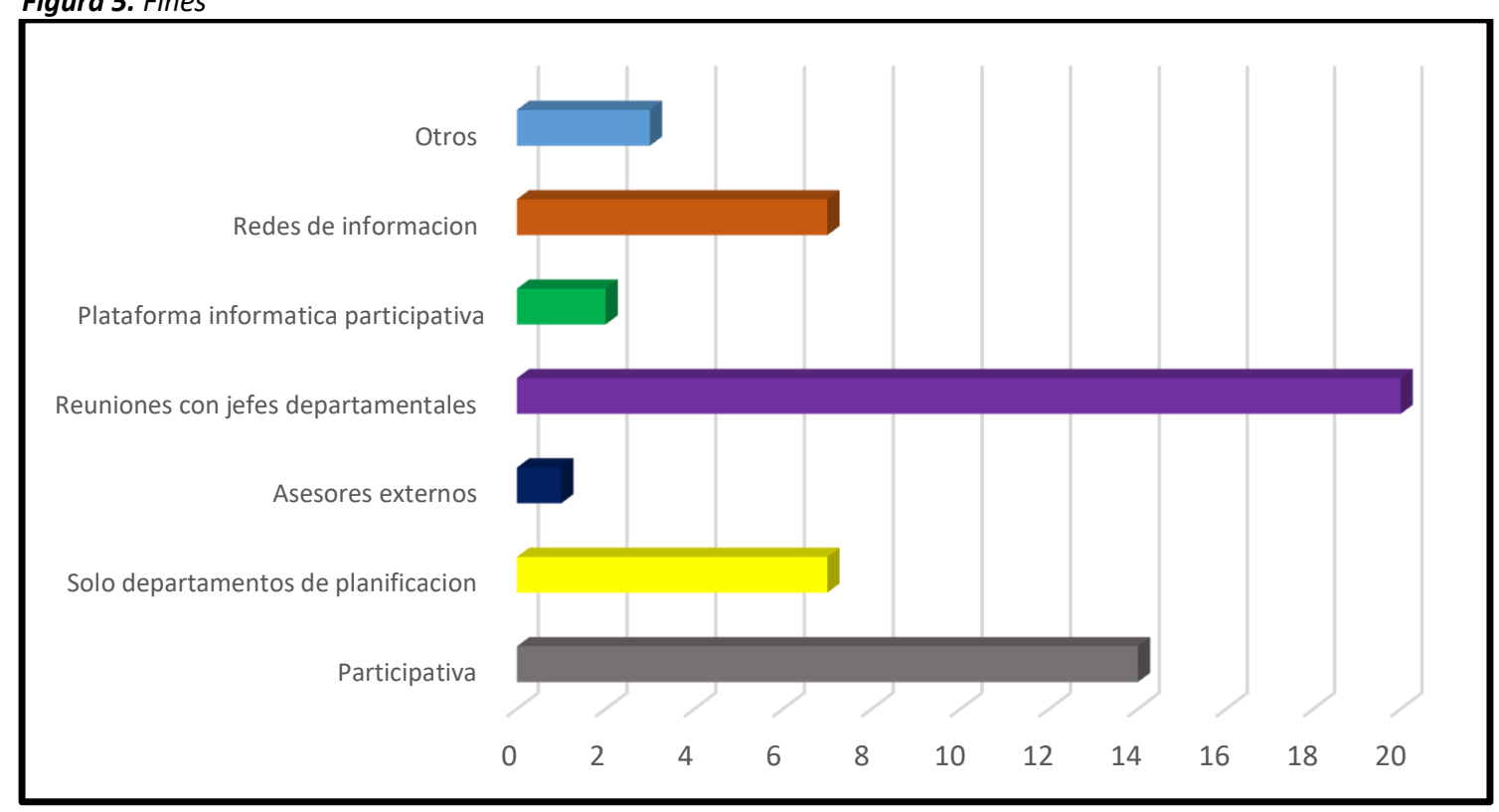

Fuente: Autores.

\section{Metodología}

Referente a la metodología que utilizan las instituciones públicas para la planificación el $37 \%$ lo hacen en reuniones con jefes departamentales, el $26 \%$ de forma participativa (interna y externa), el $13 \%$ utiliza redes de información, 5\% por plataforma informática participativa, $4 \%$ otro tipo de metodología como grupos de interés, encuestas o simplemente la realiza el departamento de planificación; y, el $2 \%$ contrata asesores externos. 


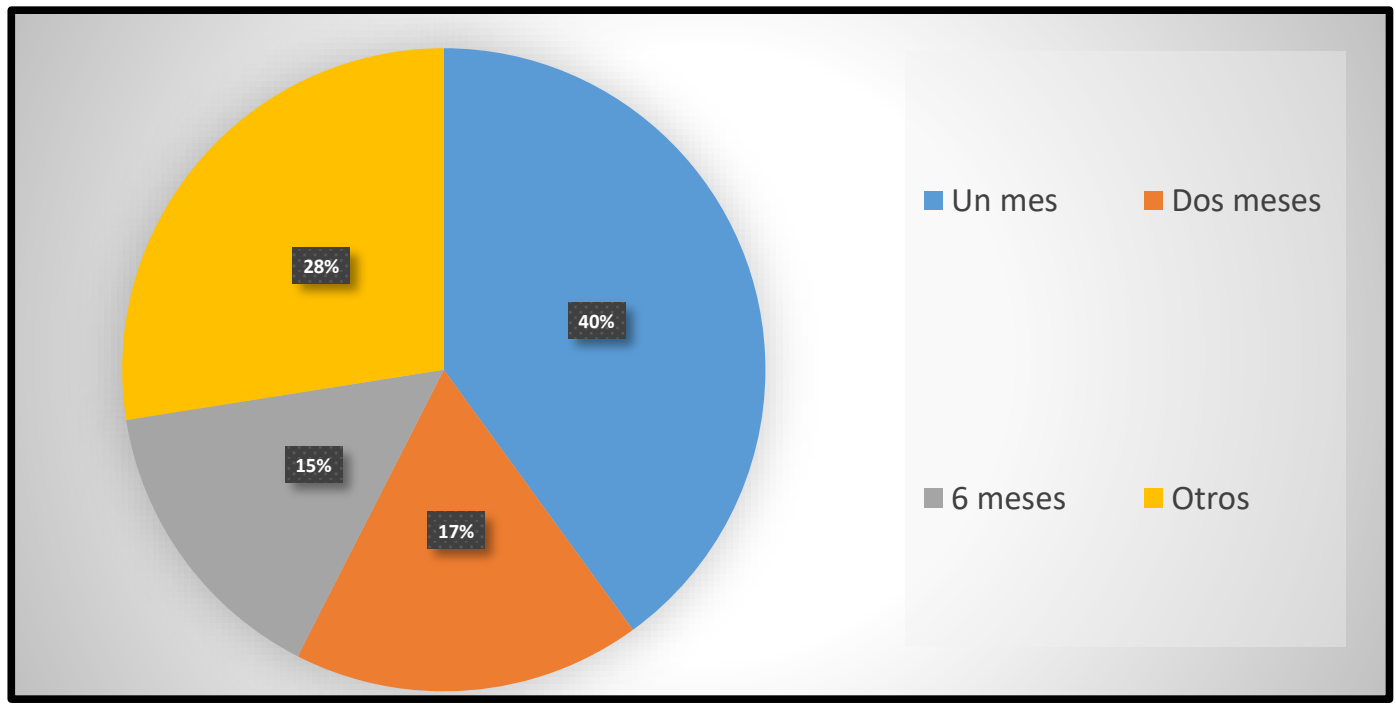

Fuente: Autores.

\section{Tiempo que se demora para realizar la planificación estratégica}

El $40 \%$ manifestaron que el tiempo estimado para realizar su planificación es de un mes, el $17 \%$ corresponde a dos meses y con un $15 \% 6$ meses; mientras que el $28 \%$ otros periodos indicando que el lapso es superior a 6 meses.

\section{Medios de socialización}

El $45 \%$ de las instituciones públicas utilizan sus páginas web y software como Quipux para socializar sus planes, el $32 \%$ que se ha definido como otros se refiere a espacios en radios o reuniones de trabajo, mientras que el $13 \%$ utilizan el método tradicional a través de carteleras y, el $6 \%$ realiza esta actividad mediante folletos y un $4 \%$ no socializa su información.

Figura 7. Medios para socializar la planificación

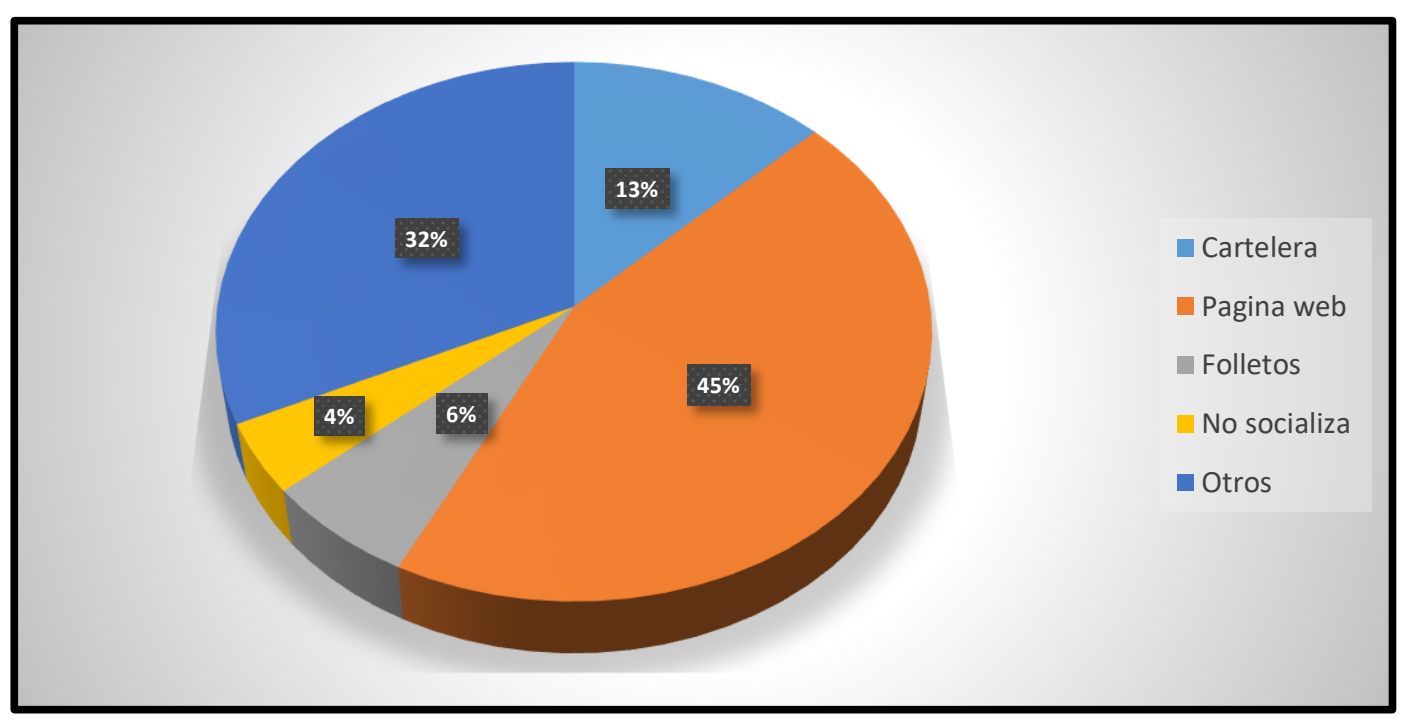

Fuente: Autores.

\section{Elaboración de presupuestos}

Respecto a la distribución del presupuesto el $25 \%$ la realizan por departamentos, el $37 \%$ por proyectos, el $5 \%$ sostuvo que la distribución se realiza en base a prioridades del entorno, el $3 \%$ de acuerdo a los resultados obtenidos en el periodo anterior y por ultimo un $2 \%$ por inversiones y el $28 \%$ de otras maneras. 
Figura 8. Distribución de presupuesto

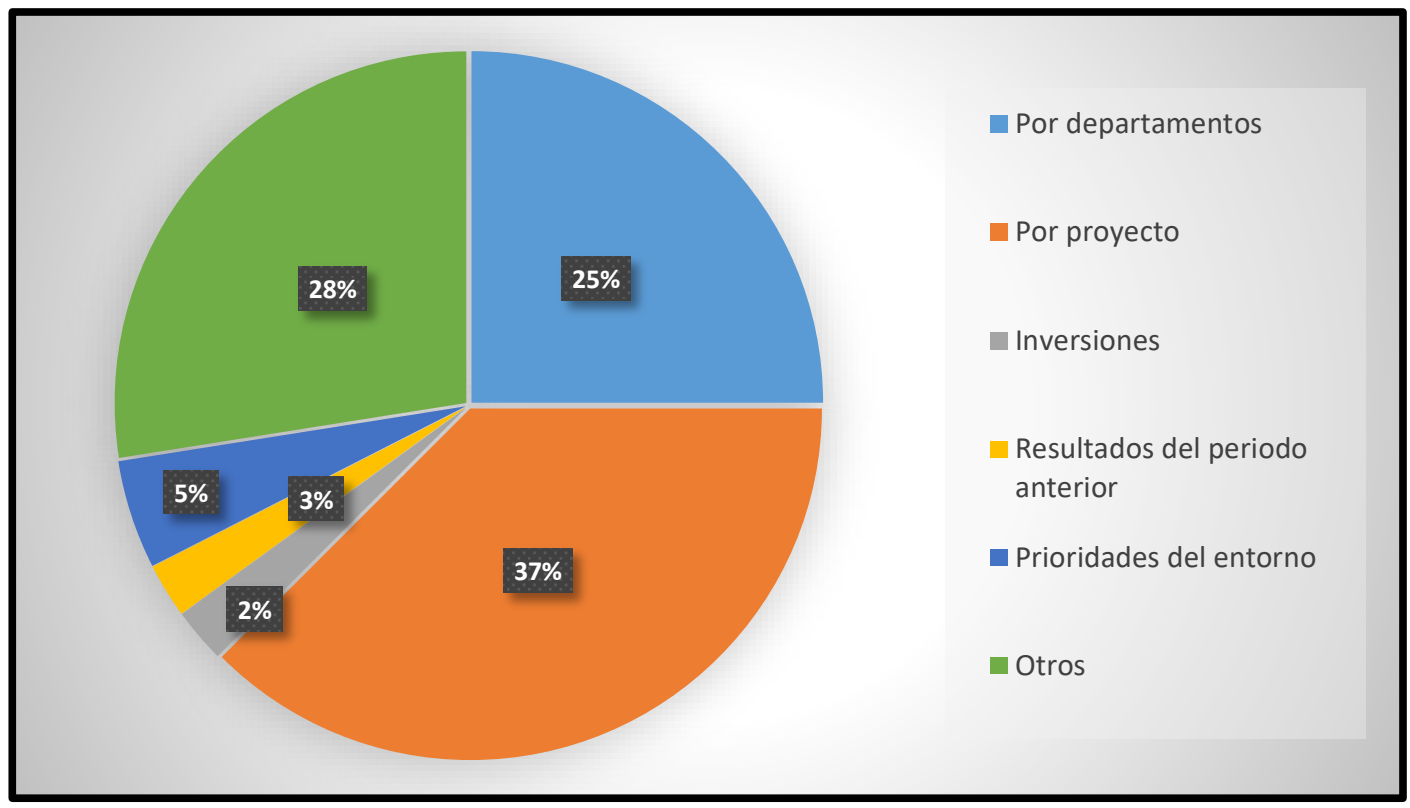

Fuente: Autores.

Tiempo de seguimiento del plan anual de políticas públicas (PAPP)

El $23 \%$ de los encuestados sostuvieron que el seguimiento de la planificación se realiza mensual, $23 \%$ semestral, el $21 \%$ trimestral, $10 \%$, cuatrimestral, el $7 \%$ de manera continua y el $16 \%$ desconocía del tema.

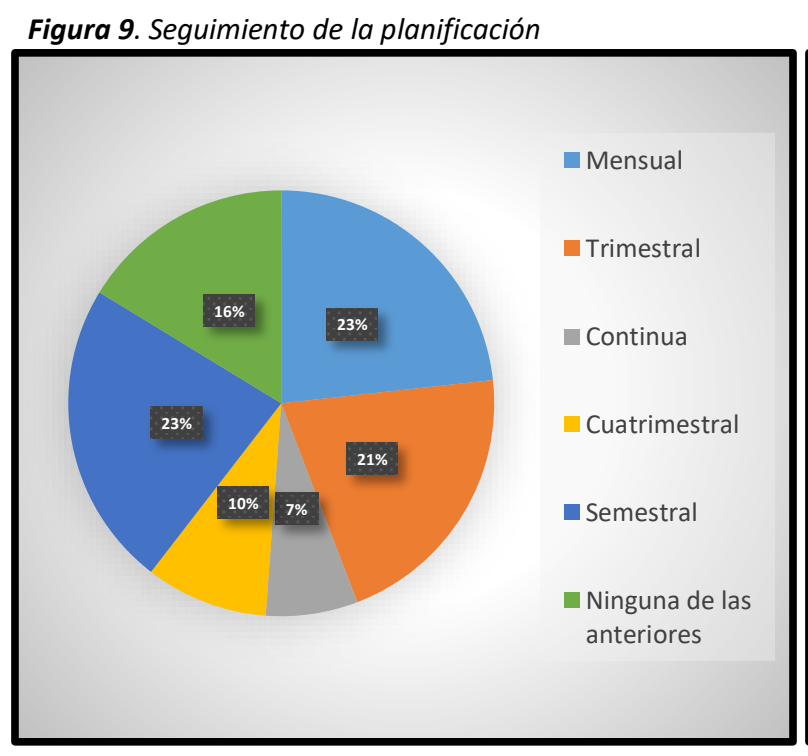

Fuente: Autores.

\section{Tipo de control y seguimiento de su planificación}

El $66 \%$ realiza el seguimiento y control de la planificación mediante el desempeño de los trabajadores, el $12 \%$ realiza seguimientos en
Figura 10. Control y seguimiento

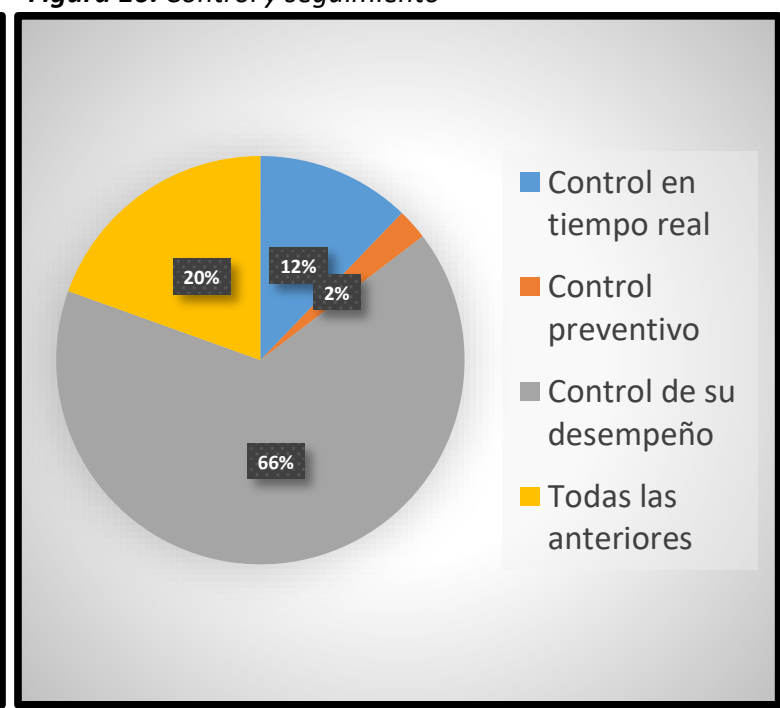

Fuente: Autores

tiempo real, el $2 \%$ realizaba controles preventivos y el $20 \%$ aplica de acuerdo a la situación todas los tipos de control antes mencionados. 


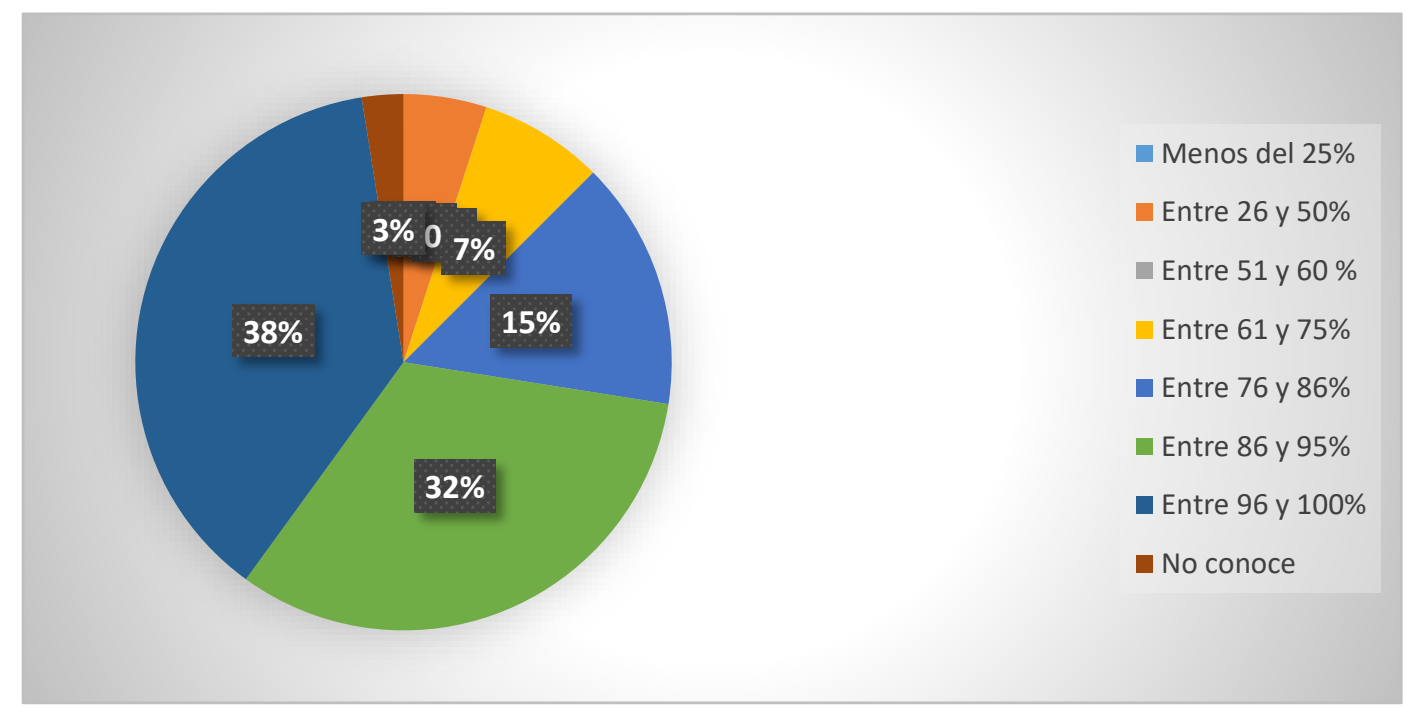

Fuente: Autores.

\section{Porcentaje de cumplimiento del plan institucional del año anterior}

El $32 \%$ de los encuestados respondieron que el cumplimiento del plan institucional estaba entre el 96 y $100 \%$, el $38 \%$ entre 76 y $96 \%$; el $15 \%$ está por debajo del $25 \%$, el $15 \%$ entre el 26 $50 \%$ y el $3 \%$ desconocían el tema.

\section{Porcentaje de ejecución presupuestaria del año anterior}

El 38 de los encuestados ejecuto su presupuesto entre el 86 y $95 \%$, mientras que el
$30 \%$ logro ejecutar entre el 96 y $100 \%$ de su presupuesto, el $11 \%$ de los encuestados desconocen del tema, el resto de los encuestados ejecutaron su presupuesto por debajo del $60 \%$.

Figura 12. Presupuesto ejecutado

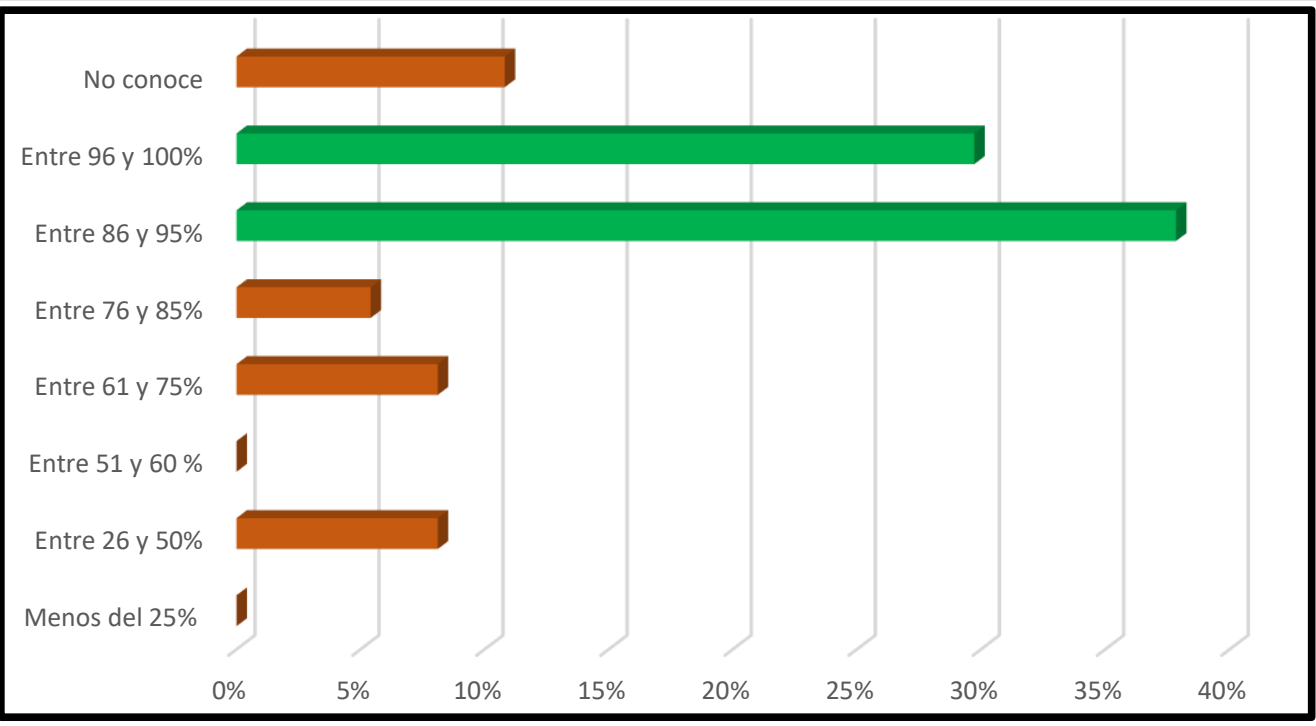

Fuente: Autores. 
Figura 13. Tiempo de socialización de la ejecución de la planificación

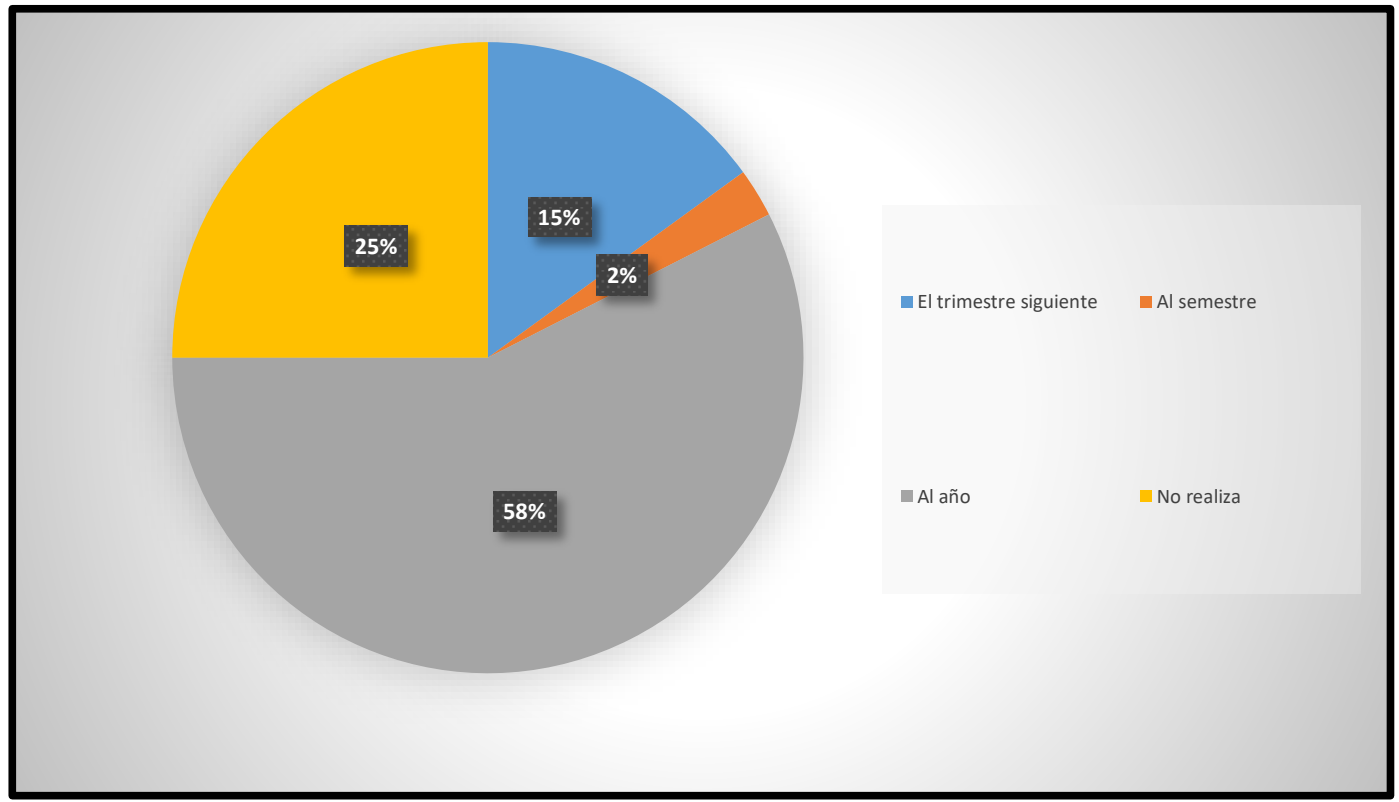

Fuente: Los autores.

\section{Tiempo de socialización de informes}

El $58 \%$ socializa al año la ejecución de su planificación, el $2 \%$ cada 6 meses, el $25 \%$ no realiza, mientras que el $15 \%$ cumple al trimestre siguiente de cada año.

\section{Medios que se utilizan para la rendición de} cuentas de la comunidad.

El $28 \%$ publica los resultados obtenidos para parte de la rendición de cuentas a través de su

Figura 14. Medios para rendición de cuentas

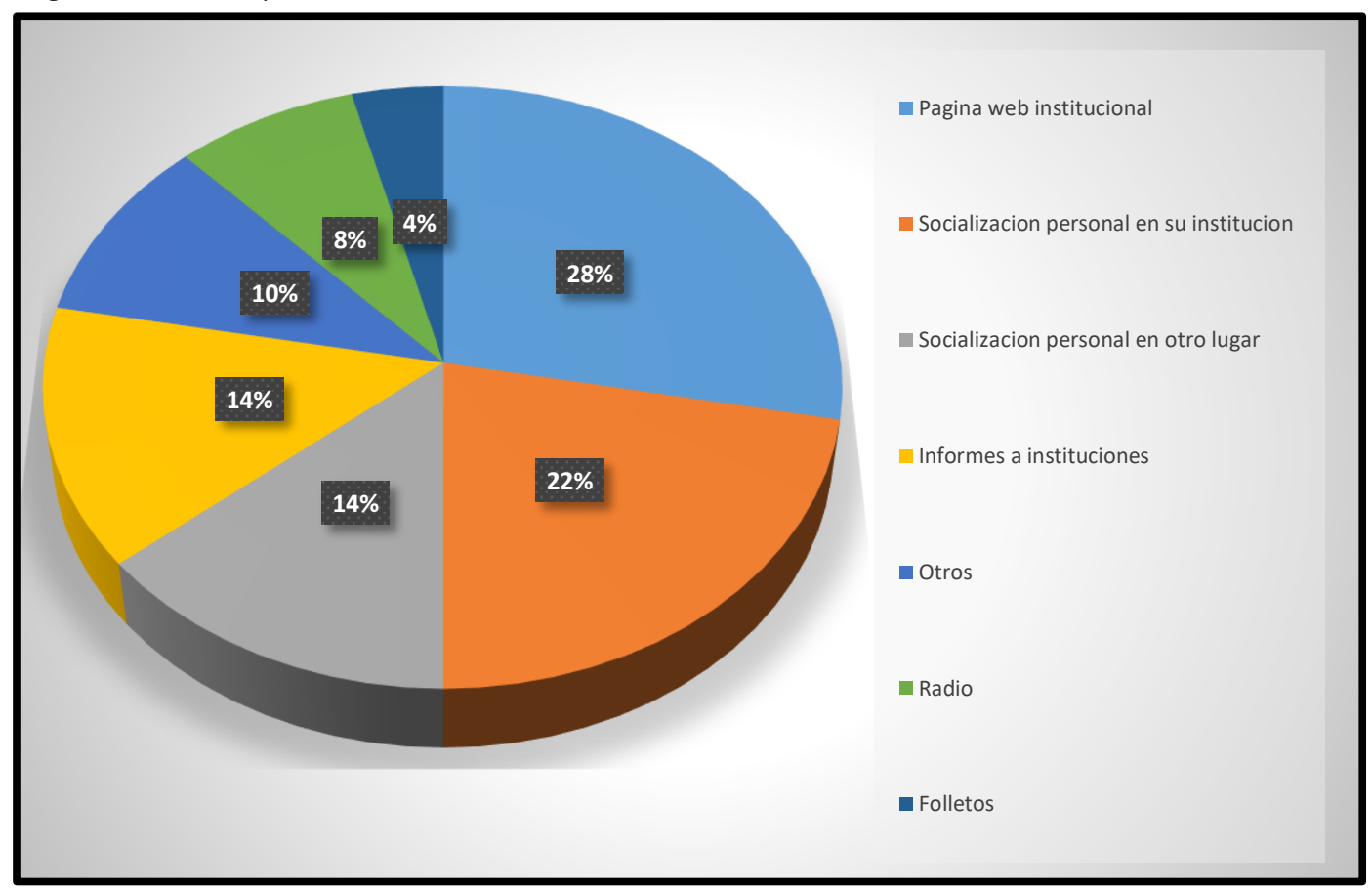

Fuente: Los autores. página web institucional, el $22 \%$ lo socializa con el personal de la misma institución, el 14\% de los encuestados usan informes institucionales y socializaba en otro lugar, el $10 \%$ usaba otro tipo de medios como reuniones o revistas, el $8 \%$ utiliza la radio y finalmente el $4 \%$ lo realizaba mediante folletos. 
Figura 15. Quienes realizan seguimiento a la planificación

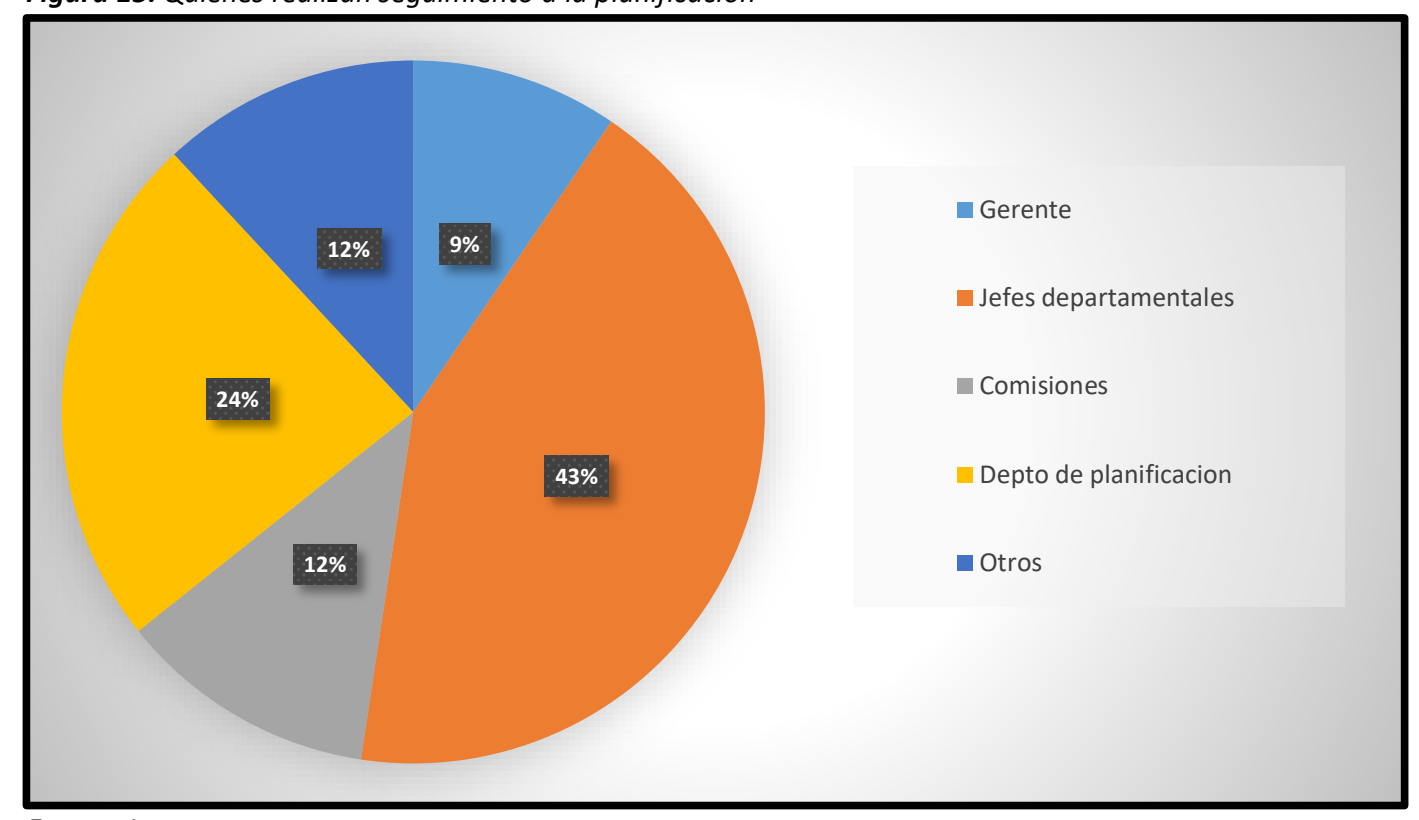

Fuente: Los autores.

\section{Quiénes realizan el seguimiento del cumplimiento del plan estratégico en la empresa}

El $43 \%$ los jefes departamentales realizan el seguimiento del plan estratégico, el $24 \%$ es ejecutada por el departamento de planificación, el $12 \%$ conforma una comisión para el seguimiento, y en el $9 \%$ de las empresas el gerente se encarga de esta función.

\section{Discusión}

\section{Planificación estratégica}

La planificación estratégica nos indica las acciones a emprender para conseguir los fines; los planes no sirven para nada: lo que si merece la pena es planificar (Romero, 2010). En este sentido, podemos decir que los administradores de las instituciones públicas de Santa Elena en su totalidad reconocen la etapa de la planificación, pero a medida que se avanza con el análisis, se evidencia que lo hacen por cumplir con el proceso de adquisición de recursos económicos por parte del Estado, lo correcto es ver la planeación como una herramienta que tiene la finalidad de crear bases y establecer el rumbo de una empresa creando estrategias para cumplir con la visión, misión y objetivos, tal como menciona (Peteraf, 2012). Resulta incorrecto pensar en la construcción de un plan estratégico institucional para cumplir con una formalidad o un estándar de calidad, la planificación representa tomar una decisión hoy para consecuencias futuras a través de un proceso debidamente monitoreado.

El proceso de ejecución de la planificación estratégica no solo se trata de conocer la misión, visión y filosofía; o de establecer objetivos y metas a través de estudios prospectivos que se ejecutan por medio de programas y proyectos, sino que se fundamenta en el análisis profundo de identificar la situación actual y real para establecer el punto de partida, para ello es necesario contar con los insumos como son: la evaluación de la misión y visión, diagnóstico situacional, informes de años anteriores y la determinación de recursos; no se puede planificar sin conocer la razón de ser y hasta donde aspira llegar la empresa, tampoco el desconocer las amenazas y oportunidades que tiene, reflexionar lo que ha logrado y con qué recursos ha contado para posteriormente continuar, la falta de insumos en la práctica de la planificación de instituciones públicas disminuye su importancia y utilidad.

Es muy importante diferenciar en su elaboración, el tiempo de construcción de un plan estratégico de desarrollo institucional (PEDI) o plan plurianual de política pública (PPPP) y el Plan Operativo Anual (POA) o Plan Anual de Política Pública (PAPP); a pesar de que los dos documentos reflejan una planificación el impacto es muy diferente, el estratégico direcciona la empresa a través del cumplimiento de la visión, mientras que el operativo 0 anual ejecuta lo planificado estratégicamente sin dejar de lado el principio 
de flexibilidad que se aplica al evaluar su cumplimiento o en los seguimientos, en la mayoría de las empresas públicas se desconoce o no se aplica esta diferenciación, en los dos casos se ha manifestado que el tiempo de construcción es un mes, tiempo muy forzado en el plan estratégico considerando que debe realizarse utilizando la metodología participativa. Por lo cual se entiende la poca importancia que se da a la planificación reflejada en los periodos de ejecución y en los porcentajes de cumplimiento.

\section{Responsabilidad de la planificación estratégica de las instituciones públicas}

Para cumplir con la misión de las instituciones públicas en la que prevalece la satisfacción de necesidades de la población y la calidad del servicio prestado, es necesario ejecutar una gestión pública eficaz que no se base en un simple discurso político sino en asumir la responsabilidad de representante legal; esto implica fortalecer y formar parte del tejido social (empresa privada, habitantes, Estado) con la finalidad de lograr una planificación que oriente y direccione al logro de grandes metas en el que se evidencie el desempeño de la fuerza laboral, la eficiencia administrativa y la consecución de los objetivos.

La responsabilidad que tienen los representantes de las instituciones públicas de la provincia de Santa Elena implica gerenciar para mejorar de manera general el desarrollo humano de 308.693 habitantes (INEC), 2010), de cumplir los compromisos propuestos y aceptados por los ciudadanos que luego juzgarán o reconocerán el impacto social de la población. Es importante destacar que desde el año 2007 se ha recuperado la planificación en el sector público y en la actualidad se mantiene con el Plan de Desarrollo Nacional 2017-2021 realizada por la Secretaría Nacional de Planificación y Desarrollo, planificación que direcciona a las instituciones del Estado y al que todas las organizaciones deben tributar. De acuerdo a los resultados obtenidos se ha interpretado la descoordinación del direccionamiento estratégico nacional con el regional, razón por la que la mayoría de las instituciones públicas no alcanzan una cualificación sobresaliente y justifican su socialización incumpliendo con la Ley Orgánica de Transparencia y Acceso a la información Pública.

La aplicación de la administración pública en la construcción de los planes estratégicos evalúa los roles y habilidades que tiene su representante, porque no es exclusiva y cerrada del gobierno sino que involucra a toda la comunidad simpatizantes o no, son actores que conocen los problemas y que van a exigir soluciones pero que el gobernante es quien consensa, analiza la viabilidad del plan y decide que hacer.

\section{Metodología y control}

La gobernabilidad está muy relacionada con las competencias asignadas a las organizaciones públicas en la Constitución de la República y demás normativa legal vigente (Barrios, 2011), es decir que cada institución pública tiene su competencia y de acuerdo a ello el control, por lo que el seguimiento y control no es responsabilidad de un solo departamento interno de la organización, en particular de la dependencia de planificación, sino que también hay competencia en instituciones externas como la Contraloría General del Estado y para ello están las Normas de Control Interno para el sector Público de la República del Ecuador; según la información obtenida mediante la encuesta, en muchas ocasiones los que se encargan de hacer el seguimiento del plan estratégico son los mismos gerentes con sus jefes departamentales de una forma mensual o semestral vinculada con el desempeño de los trabajadores a través de reuniones de los jefes departamentales.

La productividad de las empresas del Estado se vincula con el alto desempeño institucional relacionada siempre con la provisión de más y mejores bienes, servicios y actos de regulación públicos para los ciudadanos (Barrios, 2011) y para ello se tiene que exponer los logros, algunas lo han realizado a través de su rendición de cuentas a la comunidad, por lo general se pública en la página web de cada una de las instituciones y en otras ocasiones se socializa con el personal de la misma institución, situación que hay que mejorar.

\section{Conclusiones}

La planificación estratégica de las instituciones públicas, no aporta directamente al cumplimiento del Plan Nacional de Desarrollo, consecuencia del desconocimiento y la mala práctica en el proceso de construcción y control de lo previsto, ignorando los insumos principales como son la misión, visión, diagnóstico situacional y la determinación de recursos. Por lo que es de mucha importancia en cada cambio de autoridad establecer un proceso de acompañamiento y capacitación de los funcionarios para la elaboración de planes estratégicos y operativos, definición de estrategias, indicadores y sistemas de 
monitoreo, esto considerando que en su mayoría no tienen un perfil administrativo.

Los objetivos estratégicos a nivel nacional están vigentes desde octubre del 2017, lo que obliga a hacer uso de la flexibilidad de la planificación y ajustar los planes estratégicos y operativos de los gobiernos seccionales materializando las metas causando un efecto cascada, lo que permite evaluar la gerencia pública en todos los niveles jerárquicos.

Este trabajo evidencia las debilidades en la planificación estratégica de las instituciones del sector público de la provincia de Santa Elena y permite hacer una reflexión respecto a las competencias del monitoreo y control de su labor; como ciudadanos debemos analizar nuestro voto y vincular las propuestas con las competencias y el desarrollo local.

\section{Bibliografía}

Atencio, J. (2009). El control de gestión estratégica en las organizaciones (CICAG). Centro de Investigación de Ciencias Administrativas y Gerenciales, 6. Recuperado el 27 de 10 de 2017, de http://publicaciones.urbe.edu/index.php/cicag/article/viewAr ticle/482/1194: http://publicaciones.urbe.edu/index.php/cicag/article/viewArticle/4 $82 / 1194$

Barrios, A. Z. (2011). Planificación estratégica, presupuesto y control de la gestión pública. Caracas: UCAB.

CUENTAS, N. D. (2017). Plan General de Contabilidad 3. a edición 2017. Madrid: Ediciones Paraninfo, S.A.

INEC), I. N. (2010). Resultado del Censo 2010 de poblacion y vivienda en el Ecuador. Santa Elena. Recuperado el 12 de Noviembre de 2017, de http://www.ecuadorencifras.gob.ec/wp-content/descargas/Manulateral/Resultados-provinciales/ santa_elena.pdf

Jiménez, M. P. (2014). Diccionario de Administración y Finanzas. Estados Unidos de America: Palibrio LLC.
López, F. (2009). La empresa explicada de forma sencilla. Catalunya: Libros de cabecera S.L.

Marjorie Rodríguez C. (20 de Abril de 2016). Incidencia del control de la gestión administrativa de bienes muebles de la empresa municipal EMASA - EP, del cantón Santa Elena, Provincia de Santa Elena, Año 2015". Libertad, Santa Elena. Recuperado el 12 de Noviembre de 2017, de http://repositorio.upse.edu.ec/bitstream/460 00/3242/1/UPSE-TAP-2015-0059.pdf

Pérez, J., \& Gardey, A. (2014). Definición.DE. Obtenido de https://definicion.de/sectorpublico/

Perez, V. (16 de 11 de 2017). Plan operativo anual. Ayuntamiento de Tudela. Obtenido de file:///C:/Users/maria/Downloads/ Documento.pdf

Peteraf, T. I. (2012). Administración estratégica. Mexico: Mc Graw Hill.

Romero, A. F. (2010). Dirección y planificación estratégica en las empresas $y$ organizaciones. España: Ediciones Diaz y Santos. Obtenido de https://books.google.es/books?id=_LnnKTP $\mathrm{ZONgC \& printsec}=$ frontcover $\& \mathrm{hl}=\mathrm{es} \# \mathrm{v}=\mathrm{one}$ page $\& q \& f=f a l s e$

Sainz, J. M. (2015). El plan estratégico en la practica . Madrid: ESIC EDITORIAL.

Sala, P. G. (2015). Gestión de un pequeño comercio (2015). Madrid: Editex.

Sergio, V. (12 de Noviembre de 2017). GESTION.ORG. Obtenido de GESTION.ORG:

https://www.gestion.org/recursoshumanos/gestion-competencias/1770/elcontrol-de-la-gestion-estrategica/

Tobar, I. H. (s.f.). La Administración pública en el Ecuador. Informativo ESPAE. Recuperado el 12 de noviembre de 2017, de https://www.dspace.espol.edu.ec/bitstream/ 123456789/6650/3/Administraci\%C3\%B3n \%20P\%C3\%BAblica\%20en\%20el\%20Ecua dor.pdf 